\title{
Rapid bedside diagnosis of pyomyositis with ultrasound-guided needle aspiration
}

\author{
Arash Kohanteb, Diku Mandavia, Dina Seif \\ Department of Emergency Medicine, LAC+USC Medical Center, Keck University of Southern California, Los Angeles, United \\ States.
}

Correspondence: Arash Kohanteb. Address: Department of Emergency Medicine, LAC+USC Medical Center, Keck University of Southern California, Los Angeles, United States. Email: arash.kohanteb@gmail.com

Received: September 18, 2014 Accepted: October 14, $2014 \quad$ Online Published: November 6, 2014

DOI : 10.5430/ijdi.v2n1p24 URL: http://dx.doi.org/10.5430/ijdi.v2n1p24

\begin{abstract}
A 62-year-old male with a past medical history of cirrhosis presented to the emergency department with left thigh pain and swelling 2 weeks after trauma to the left lower extremity due to a ground level fall. On arrival, physical exam revealed significant edema, erythema and warmth of the left thigh concerning for infection. Point-of-care soft tissue ultrasound by the emergency physician revealed multiple complex fluid collections deep within the quadriceps muscle. Ultrasoundguided needle aspiration of these fluid collections was performed at the bedside while waiting for surgical consultation and MRI. Copious amounts of purulent fluid were aspirated prompting the early diagnosis of pyomyositis. Therapeutic management was expedited as a result of these findings. Point-of-care ultrasound is a valuable tool for the early and rapid detection of pyomyositis and can expedite definitive treatment.
\end{abstract}

\section{Keywords}

Point of care ultrasound, Pyomyositis, Abscess, Ultrasound-guided aspiration, Emergency ultrasound, Emergency medicine

\section{Introduction}

Pyomyositis, which is the presence of an abscess or multiple abscesses within skeletal muscle is typically a disease of the tropics but has recently become more prevalent in temperate climates ${ }^{[1]}$. In the United States, HIV-negative patients who develop pyomyositis frequently have a predisposing medical condition. In temperate climates it is associated with underlying conditions such as diabetes, malignancy, HIV infection and chronic liver disease ${ }^{[2,3]}$. Blunt trauma as well as muscle overuse injuries is among other etiologies of pyomyositis, often involving hematomas that become infected ${ }^{[4]}$. Primary pyomyositis most commonly affects the quadriceps, gluteal and iliopsoas muscles and occurs in three consecutive stages: diffuse muscle infection, abscess formation, and sepsis ${ }^{[6]}$. Physical exams often overlap with less emergent pathology, including cellulitis, hematoma or superficial abscess and a discrete abscess may not always be palpable ${ }^{[2]}$. Traditionally, MRI is the preferred diagnostic tool. The fluid collections of pyomyositis can be seen on MRI as hyperintense signals on T2 weighted images. In situations where MRI is not readily available or patients are too unstable, it can be difficult to make the diagnosis and a delay in diagnosis can lead to sepsis and death ${ }^{[6]}$. 
Ultrasound imaging at the bedside and ultrasound-guided needle aspiration can expedite diagnosis, prompt antibiotic coverage and definitive surgical treatment, which may lead to better patient outcomes ${ }^{[4]}$.

\section{Case presentation}

A 62-year-old male with a past medical history of liver cirrhosis presented to the Emergency Department (ED) with a chief complaint of left leg pain and swelling for two weeks after sustaining a ground level fall. The patient was seen one week prior with X-rays showing no evidence of fracture or bony abnormality. The patient stated that his leg pain had become so severe that he was no longer able to ambulate. The patient denied fever, chills, shortness of breath, chest pain or any motor or sensory deficits.

On physical examination the patient was alert and oriented but was ill-appearing. His initial vital signs were blood pressure 120/64 mm Hg, heart rate 86 beats per minute, respirations 20 per minute, oxygen saturation of $97 \%$ on room air and temperature 97.0 degrees Fahrenheit. The patient was noted to have erythema, warmth and dramatic 4+ pitting edema extending from the left foot to the left groin. The entire left lower extremity was tender to palpation. A left knee effusion was present but there was no pain with movement of the knee. Sensation and pulses were intact in all extremities. Of note, no palpable abscess, open wounds, bullae or crepitus were present. The cardiopulmonary exam was normal and abdominal exam revealed a firm liver edge. Additional physical exam findings included scleral icterus and asterixis.

The laboratory results were significant for white blood cell count $26,100 / \mathrm{mm}^{3}$ with profound neutrophilia, CRP $117.6 \mathrm{mg} / \mathrm{dL}$, lactate $6.0 \mathrm{mg} / \mathrm{dL}$, platelets 134,000/. $\mathrm{mm}^{3}$, hemoglobin $10.4 \mathrm{~g} / \mathrm{dL}$, INR 3.0, ammonia $93 \mathrm{mcg} / \mathrm{dL}$, creatinine $4.01 \mathrm{mg} / \mathrm{dL}$ and BUN $115 \mathrm{mg} / \mathrm{dL}$. CXR showed no acute abnormalities.

Bedside ultrasound was performed by the Emergency Physician (EP) using a 13-6 MHz high-frequency linear array transducer (SonoSite FujiFilm, Bothell, Washington, USA), showing an extensive fluid collection within the muscle extending from the knee to the upper thigh (see Figure 1 and Figure 2). A large knee effusion was also visualized on bedside US (see Figure 3). Limited compression ultrasonography was also performed by the EP to evaluate the common femoral and popliteal veins for deep vein thrombosis (DVT) and was found to be negative ${ }^{[7]}$. Of note, a radiologyperformed duplex ultrasound of the lower extremity was later performed and confirmed no evidence of DVT but multiple fluid collections without evidence of gas were seen and were interpreted as hematomas.

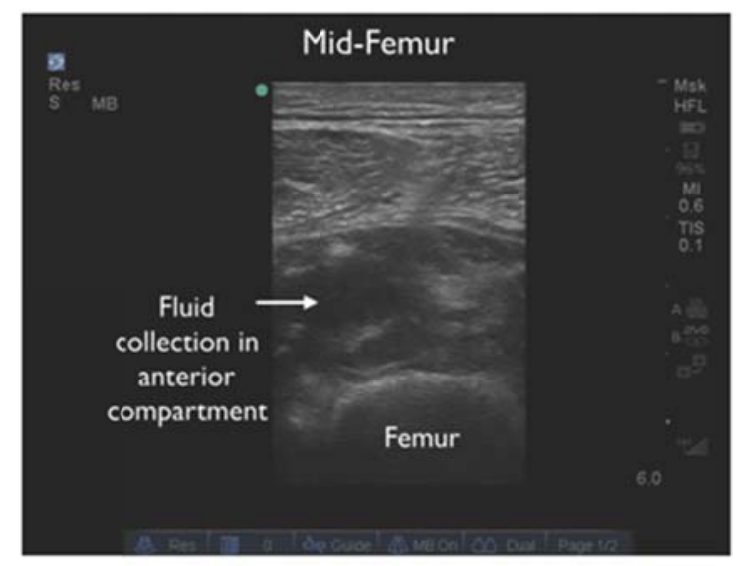

Figure 1. Transverse view of left mid-thigh shows complex fluid collection seen within muscles of anterior compartment

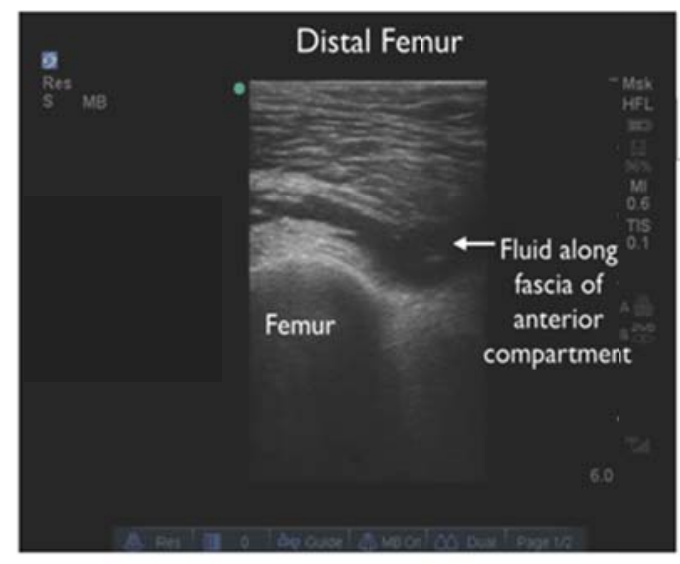

Figure 2. Transverse view of distal left thigh shows complex fluid collection seen within muscles of anterior compartment 


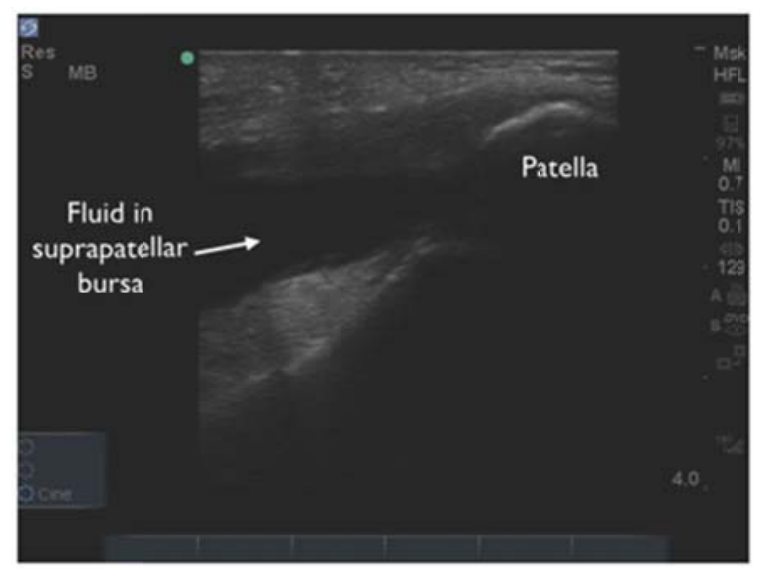

Figure 3. Sagittal view of fluid collection seen in supra-patellar bursa

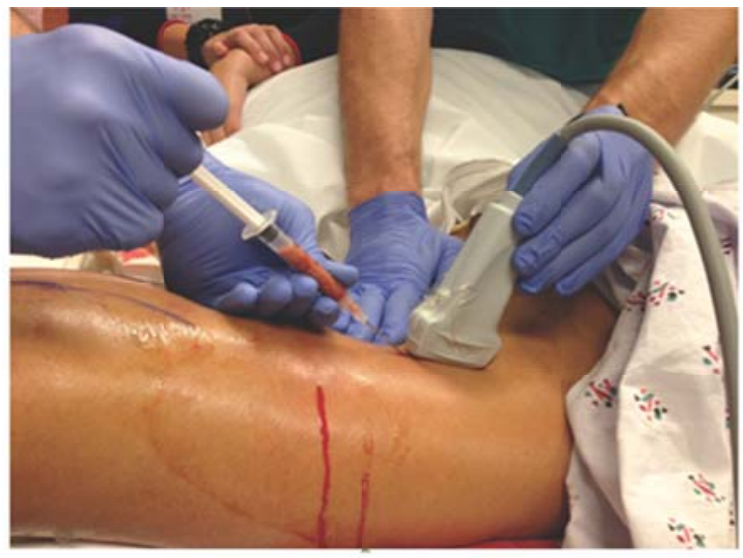

Figure 4. In-plane ultrasound-guided aspiration of pus from proximal thigh

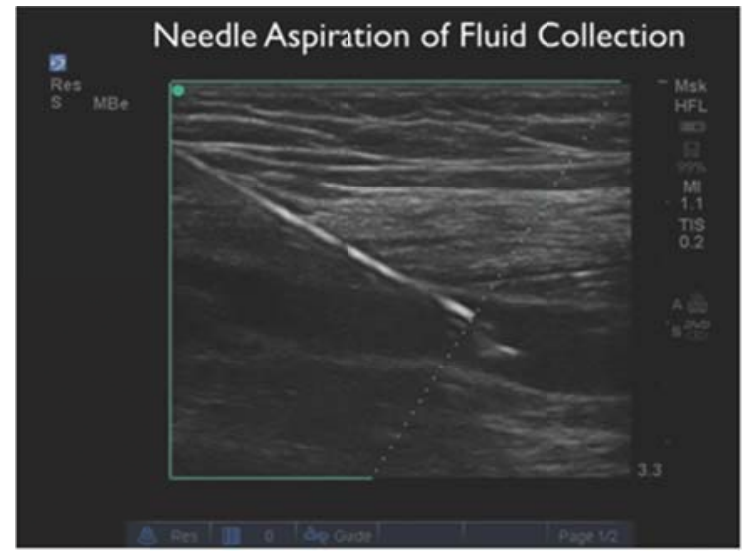

Figure 5. Advanced needle visualization of in-plane ultrasound-guided aspiration of pus

Intravenous antibiotics were administered for cellulitis early in the ED course. After the visualization of extensive fluid collections on bedside ultrasound, treatment was extended to include broad-spectrum coverage and the orthopedic surgery service was consulted. The initial recommendations were to continue antibiotics and obtain an MRI of the extremity to further characterize the fluid collections.

While awaiting advanced imaging and further surgical recommendations, the patient's vital signs deteriorated. He became hypothermic and hypotensive, requiring aggressive resuscitation. At this point it was decided by the EP to perform an ultrasound-guided aspiration of the fluid collection to determine whether the fluid seen was hematoma or the source of sepsis. Using an 18-gauge spinal needle, the most superficial fluid collection was aspirated in a sterile fashion ${ }^{[8]}$. An in-plane technique was employed using Advanced Needle Visualization (ANV) mode (see Figure 4 and Figure 5). Approximately 20 milliliters of purulent material was aspirated from the anterior thigh just deep to the muscle fascia and was sent for culture and gram stain. The orthopedic surgery service was re-consulted for pyomyositis and emergent open washout in light of the aspiration results. MRI was expedited to map out the surgical approach for debridement and the patient was taken emergently to the operating room.

Hospital course: The MRI findings showed a complex fluid collection within the anterior compartment of the left thigh measuring $6.5 \mathrm{~cm} \times 9 \mathrm{~cm} \times 32 \mathrm{~cm}$ as well as fluid collections along the superficial fascia of anterior and posterior compartments of the left thigh and left distal lower extremity. In the operating room, multiple pockets of purulence were found in the anterior thigh compartment. These pockets tracked posteriorly behind the femur. Of note, the fascia was intact 
and viable. After the pockets were evacuated and copiously irrigated with saline and antibiotic fluid, five drains were left in place and the wounds were closed. A washout of the left knee was also performed. Wound cultures grew oxacillinresistant staph aureus (ORSA). The patient remained intubated after the OR and subsequently developed multiorgan system failure and ultimately the decision was made by family to withdraw care.

\section{Conclusion}

This case describes a complicated presentation of left lower extremity pyomyositis with dynamically evolving sepsis. The pathophysiology and presentation of the disease can make it difficult to diagnose. Other conditions such as DVT, cellulitis, myositis or muscular hematoma may have similar presentations adding to the diagnostic difficulty ${ }^{[2]}$. The presentation of pyomyositis occurs in a three stage process. The first stage is limited to pain and edema of the muscle. During the second stage there is abscess formation which is typically at day 10-21. The third stage is characterized by septicemia, multiple abscess formation and multiorgan dysfunction. Ninety percent of the diagnoses are made during the second stage however the presentation during this second stage can be easily mistaken for a superficial infection ${ }^{[3]}$. Although pyomyositis most commonly effects the quadriceps, gluteal and iliopsoas muscles, it can be also be found in various other muscle groups within the body ${ }^{[9]}$. Bacterial pyomyositis is most commonly cause by Staphylococcus aureus although other organisms such as Streptococcus, gram negative organisms, anaerobes and mycobacteria can be implicated based on underlying medical conditions ${ }^{[3]}$.

CT and MRI have long been the standard imaging modalities for deep tissue infections. However, in an environment where advanced imaging is difficult to obtain and can delay diagnosis and definitive treatment, bedside ultrasound and ultrasound-guided needle aspiration can rapidly establish the diagnosis. Aspiration and culture of pus remains the standard diagnostic criterion once a fluid collection has been identified. Soft tissue ultrasound can aid in initial screening by revealing potentially unexpected findings such as deep abscesses that are difficult to appreciate by physical exam alone. Furthermore, ultrasound can be performed at the bedside during active resuscitation of unstable patients, while CT and MRI require the patient to be moved out of the monitored treatment area.

Limitations of bedside ultrasound exist when compared to advanced imaging. The amount, extension and exact locations of infection may not be fully evaluated. Additionally, ultrasound cannot delineate the content of a fluid collection. However, bedside ultrasound can evaluate whether a fluid collection is superficial or deep and ultrasound-guided needle aspiration can quickly determine the content of such fluid collection.

In this case, point-of-care emergency ultrasound quickly identified intramuscular fluid collections. The initial impression of cellulitis was quickly ruled out, aggressive treatment was initiated and definitive surgical intervention was expedited by bedside ultrasound and ultrasound-guided needle aspiration. MRI ultimately supported the ultrasound findings but was unable to definitively make the diagnosis. It was not until ultrasound-guided needle aspiration was performed that the diagnosis was confirmed which then expedited definitive surgical management. Point-of-care ultrasound is a valuable tool to both identify deep soft tissue fluid collections and to aspirate them, thereby rapidly establishing the diagnosis at the bedside.

\section{Conflicts of interest}

Diku Mandavia is an employee of Fujifilm SonoSite Inc. Dina Seif has provided educational consultation for SonoSite.

\section{References}

[1] Bitoti C, Mainak M, Atri C, et al. Tropical Pyomyositis. N Am J Med Sci. 2013; 5(10): 600-03. http://dx.doi.org/10.4103/1947-2714.120796 
[2] Agarwal V, Chauhan S, Gupta RK. Neuroimaging Tropical Disease Pyomyositis. Neuroimaging Clinics of North America. 2011; 21(4): 975-83. http://dx.doi.org/10.1016/j.nic.2011.07.011

[3] Crum NF. Bacterial pyomyositis in the United States. Am J Med. 2004; 117: 420-8. http://dx.doi.org/10.1016/j.amjmed.2004.03.031

[4] Kumar MP, Seif D, Perera P, et al. Point-of-care Ultrasound in diagnosing pyomyositis: A report of three cases. J Emerg Med. 2014; pii: S0736-4679(14)00176-0 (Epub ahead of print)

[5] Stevens DL, Bisno AL, Chambers HF, et al. Practice guidelines for the diagnosis and management of skin and soft-tissue infections. Infect Dis. 2005; 41(10): 1373-1406. http://dx.doi.org/10.1086/497143

[6] Anand AK, Gopalakrishnan C, Jesuraj M, et al. Pyomyositis. Postgrad Med J. 2013; 89: 179-180. http://dx.doi.org/10.1136/postgradmedj-2012-131162

[7] Poppiti R, Papinocolau G, Perese S. Limited B-mode venous scanning versus complete color flow duplex venous scanning for detection of proximal deep venous thrombosis. J Vasc Surg. 1995; 22: 553-7. http://dx.doi.org/10.1016/S0741-5214(95)70037-4

[8] Barr L, Hatch N, Roque PJ, et al. Basic ultrasound-guided procedures. Crit Care Clin. 2014; 30(2): 275-304. http://dx.doi.org/10.1016/j.ccc.2013.10.004

[9] Bickels J, Ben-Sira L. Kessler A, et al. Primary Pyomyositis. J Bone Joint Surg Am. 2002; 84-A: 2277-86. 